\title{
Laparoscopic Total Gastrectomy and D2 Lymphadenectomy
}

\author{
Giovanni Dapri, MD, Jacques Himpens, MD, and Guy-Bernard Cadière, MD, PhD \\ Department of Gastrointestinal Surgery, European School of Laparoscopic Surgery, Saint-Pierre University Hospital, \\ Brussels, Belgium
}

\begin{abstract}
Background. Lymphadenectomy and esophagojejunostomy are considered demanding steps of laparoscopic gastrectomy for cancer. We report a laparoscopic total gastrectomy and D2 lymphadenectomy performed with a circular mechanical esophagojejunostomy.

Methods. A 51-year-old woman sought care for a degenerate gastric ulcer without other symptoms. Preoperative workup showed a voluminous ulcer of $3 \mathrm{~cm}$ at the incisura angularis with irregular margins and no satellite lymph nodes/distant metastases. Poorly differentiated adenocarcinoma was confirmed by biopsy. Five trocars were placed in the abdomen. The gastrocolic ligament close to the transverse colon was opened, and the entire greater omentum was removed en bloc. Lymphadenectomy of stations 7,8 , and 9 started by the dissection of the anterior peritoneal sheet at the pancreatic head. The proximal duodenum was sectioned by firing a linear stapler blue load with Gore SeamGuard ${ }^{\circledR}$ (W.L. Gore \& Associates, Inc, Flagstaff, AZ). The lower esophagus was partially opened by scissors, a $25-\mathrm{mm}$ circular anvil was inserted, and a
\end{abstract}

This paper was presented as video presentation during the 95th Annual Clinical Congress of the American College of Surgeons, Chicago (IL, US), October 11-15, 2009.

Electronic supplementary material The online version of this article (doi:10.1245/s10434-010-0936-0) contains supplementary material, which is available to authorized users.

(C) Society of Surgical Oncology 2010

First Received: 22 November 2009;

Published Online: 3 March 2010

G. Dapri, MD

e-mail: giovanni@dapri.net purse string was tied after complete section of the viscera. A bowel limb reaching the esophagus without tension was searched from the Treitz angle, and the anastomosis was performed. Alimentary limb of $60 \mathrm{~cm}$ was measured, and a side-to-side linear mechanical jejunojejunostomy was performed. Closure of the mesenteric defect (between biliary and common limbs) and the Petersen's space (between transverse mesocolon and alimentary limb) ended the procedure. The specimen was retrieved by suprapubic incision.

Results. Total operation time was 330 minutes. Blood loss was $200 \mathrm{~mL}$. The patient was discharged 7 days after surgery. Final stage of disease was pT1NOM0 undifferentiated adenocarcinoma.

Conclusions. Thanks to laparoscopy, a meticulous lymphadenectomy can be performed under excellent view. Advantages, besides rapid recovery of bowel function, include short hospitalization and better cosmesis. Circular stapler esophagojejunostomy can easily be performed by insertion of the anvil transabdominally. 